\title{
ON THE AVERAGE OF CENTRAL VALUES OF SYMMETRIC SQUARE $L$-FUNCTIONS IN WEIGHT ASPECT
}

\author{
WINFRIED KOHNEN AND JYOTI SENGUPTA
}

\begin{abstract}
It is proved that the central values of symmetric square $L$-functions of normalized Hecke eigenforms for the full modular group on average satisfy an analogue of the Lindelöf hypothesis in weight aspect, under the assumption that these values are non-negative.
\end{abstract}

\section{$\S 1$. Introduction}

Let $S_{k}$ be the space of cusp forms of even integral weight $k \geq 12$ with respect to $S L_{2}(\mathbf{Z})$. According to [8] (cf. also [6]) the central values of Hecke $L$-functions of normalized Hecke eigenforms in $S_{k}$ on average satisfy an analogue of the Lindelöf hypothesis when the weight varies.

The purpose of this note is to show a similar result for symmetric square $L$-functions, under the assumption that their central values are nonnegative.

For the proof we use a "kernel function" for the symmetric square $L$ function as given by Zagier in [10] and then proceed in a similar way as in $[5,6,8]$, exploiting the bounds for Petersson norms implied by the work of Iwaniec [4]. Note that the kernel function of [10] was used in [5] to prove some non-vanishing results for symmetric square $L$-functions inside the critical strip.

Recall that by the work of Gelbart and Jacquet [3] the symmetric square $L$-function (up to a variable shift) also is the standard $L$-function of a cuspidal automorphic form on $G L(3)$. From this point of view it is therefore quite natural and important to study the Lindelöf hypothesis (actually in all aspects) for those $L$-functions (compare [7]).

Received December 25, 2000.

2000 Mathematics Subject Classification: 11F03. 


\section{$\S 2$. Statement of result}

We let $\mathcal{F}_{k}$ be the set of normalized Hecke eigenforms in $S_{k}$. For $f \in \mathcal{F}_{k}$ we denote by $D_{f}(s)(s \in \mathbf{C})$ the symmetric square $L$-function of $f$ defined by analytic continuation of the Euler product

$$
\prod_{p}\left(1-\alpha_{p}^{2} p^{-s}\right)^{-1}\left(1-\alpha_{p} \beta_{p} p^{-s}\right)^{-1}\left(1-\beta_{p}^{2} p^{-s}\right)^{-1} \quad(\operatorname{Re}(s)>k)
$$

where $\alpha_{p}, \beta_{p}$ are defined by

$$
\alpha_{p}+\beta_{p}=a(p), \alpha_{p} \beta_{p}=p^{k-1}
$$

and $a(p)$ is the $p$-th Fourier coefficient of $f$. Recall that the modified function

$$
D_{f}^{*}(s):=2^{-s} \pi^{-3 s / 2} \Gamma(s) \Gamma\left(\frac{s-k+2}{2}\right) D_{f}(s)
$$

is invariant under $s \mapsto 2 k-1-s[9,10]$.

According to the generalized Riemann hypothesis, all the zeroes of $D_{f}^{*}(s)$ should lie on the critical line $\operatorname{Re}(s)=k-\frac{1}{2}$. In particular, since $D_{f}(s)$ is real on the real line, one would expect that $D_{f}\left(k-\frac{1}{2}\right) \geq 0$.

THEOREM. Suppose that $D_{f}\left(k-\frac{1}{2}\right) \geq 0$ for all $f \in \mathcal{F}_{k}$ and all $k$. Then

$$
\sum_{f \in \mathcal{F}_{k}} D_{f}\left(k-\frac{1}{2}\right)<<_{\epsilon} k^{1+\epsilon} \quad(k \rightarrow \infty)
$$

for any $\epsilon>0$ where the implied constant in $<<_{\epsilon}$ depends only on $\epsilon$.

Proof. Again as in [5], our starting point is Zagier's identity [10]

$$
\begin{aligned}
& \text { (1) } \sum_{f \in \mathcal{F}_{k}} \frac{D_{f}(s+k-1)}{\|f\|^{2}} \\
&=c_{k}^{-1} \cdot \frac{(4 \pi)^{s+k-1}}{\Gamma(s+k-1)}\left(\sum_{t \in \mathbf{Z}}\left(I_{k}\left(t^{2}-4, t ; s\right)+I_{k}\left(t^{2}-4,-t ; s\right)\right) L\left(s, t^{2}-4\right)\right. \\
&\left.+\frac{(-1)^{k / 2} \Gamma(s+k-1) \zeta(2 s)}{2^{2 s+k-3} \pi^{s-1} \Gamma(k)}\right) \quad(2-k<\operatorname{Re}(s)<k-1)
\end{aligned}
$$

where

$$
c_{k}:=\frac{(-1)^{k / 2} \pi}{2^{k-3}(k-1)}
$$


and $\|f\|$ denotes the usual Petersson norm of $f$. Furthermore, for $t$ an integer and $\Delta:=t^{2}-4$, in the range $2-k<\operatorname{Re}(s)<k-1$ we have put

$$
I_{k}(\Delta, t ; s):= \begin{cases}\frac{\Gamma\left(k-\frac{1}{2}\right) \Gamma\left(\frac{1}{2}\right)}{\Gamma(k)} \int_{0}^{\infty} \frac{y^{k+s-2}}{\left(y^{2}+i t y-\frac{1}{4} \Delta\right)^{k-1 / 2}} d y, & \text { if } \Delta \neq 0 \\ e^{\operatorname{sign} t \cdot \frac{\pi i}{2}(s-k)} \sqrt{\pi} \frac{\Gamma\left(s-\frac{1}{2}\right) \Gamma(k-s)}{\Gamma(k)}|t|^{s-k}, & \text { if } \Delta=0 .\end{cases}
$$

Finally

$$
L(s, \Delta):= \begin{cases}\zeta(2 s-1), & \text { if } \Delta=0 \\ L_{D}(s) \sum_{d \mid f} \mu(d)\left(\frac{D}{d}\right) \sigma_{1-2 s}\left(\frac{f}{d}\right), & \text { if } \Delta \neq 0\end{cases}
$$

where if $\Delta \neq 0$ we have set $\Delta=D f^{2}$ with $f \in \mathbf{N}$ and $D$ the discriminant of $\mathbf{Q}(\sqrt{\Delta}), L_{D}(s)$ is the associated Dirichlet $L$-function and $\sigma_{\nu}(m):=\sum_{d \mid m} d^{\nu} \quad(m \in \mathbf{N}, \nu \in \mathbf{C})$.

We now specialize (1) to the case $s=\frac{1}{2}$. Note that on the right-hand side of (1) the sum of the terms corresponding to $t= \pm 2$ in the sum over $t$ has a simple pole at $s=\frac{1}{2}$ and also the single term outside the sum over $t$ has a simple pole at $s=\frac{1}{2}$. Both poles cancel and a short calculation reveals that these terms altogether give the contribution

$$
\begin{gathered}
\lim _{s \rightarrow \frac{1}{2}}\left(2\left(\left(I_{k}(0,2 ; s)+I_{k}(0,-2 ; s)\right) \zeta(2 s-1)+\frac{(-1)^{k / 2} \Gamma(s+k-1) \zeta(2 s)}{2^{2 s+k-3} \pi^{s-1} \Gamma(k)}\right)\right. \\
=\frac{(-1)^{k / 2} \sqrt{\pi}}{2^{k-2} \Gamma(k)}\left(C_{1} \Gamma^{\prime}\left(k-\frac{1}{2}\right)+C_{2} \Gamma\left(k-\frac{1}{2}\right)\right)
\end{gathered}
$$

where $C_{1}$ and $C_{2}$ are absolute constants.

We therefore obtain from (1) that

$$
\begin{aligned}
& \sum_{f \in \mathcal{F}_{k}} \frac{D_{f}\left(k-\frac{1}{2}\right)}{\|f\|^{2}} \\
= & \frac{(-1)^{k / 2}(k-1) 2^{3 k-3} \pi^{k-3 / 2}}{\Gamma\left(k-\frac{1}{2}\right)} \\
& \times\left(\sum_{t \geq 1, t \neq 2}\left(I_{k}\left(t^{2}-4, t ; \frac{1}{2}\right)+I_{k}\left(t^{2}-4,-t ; \frac{1}{2}\right)\right) L\left(\frac{1}{2}, t^{2}-4\right)\right. \\
+ & \left.I_{k}\left(-4,0 ; \frac{1}{2}\right) L\left(\frac{1}{2},-4\right)+\frac{(-1)^{k / 2} \sqrt{\pi}}{2^{k-2} \Gamma(k)}\left(C_{1} \Gamma^{\prime}\left(k-\frac{1}{2}\right)+C_{2} \Gamma\left(k-\frac{1}{2}\right)\right)\right) .
\end{aligned}
$$


We shall now estimate the right-hand side of (2) in $k$-aspect using arguments similar to those used earlier in [5].

First of all, given $\epsilon^{\prime}>0$, for any $t \in \mathbf{Z}, t \neq \pm 2$ one has

$$
L\left(\frac{1}{2}, t^{2}-4\right)<<_{\epsilon^{\prime}}\left|t^{2}-4\right|^{1 / 2+\epsilon^{\prime}}
$$

where the constant implied in $<\epsilon_{\epsilon^{\prime}}$ only depends on $\epsilon^{\prime}$ and not on $t[2$, chap. 12, problem 22 (b)].

Also the inequalities

$$
\begin{aligned}
I_{k}\left(t^{2}-4, t ; \frac{1}{2}\right)+I_{k}\left(t^{2}-4,-t ; \frac{1}{2}\right) \\
<< \begin{cases}\left(t^{2}-4\right)^{-1 / 4}\left(\frac{t-\sqrt{t^{2}-4}}{t+\sqrt{t^{2}-4}}\right)^{\frac{k-1}{2}} \frac{\Gamma\left(k-\frac{1}{2}\right)^{2}}{2^{k} \Gamma(k)^{2}}, & \text { if } t \geq 3 \\
\frac{\Gamma\left(k-\frac{1}{2}\right)^{2}}{2^{k} \Gamma(k)^{2}}, & \text { if } t=1\end{cases}
\end{aligned}
$$

hold where the constants involved in $<<$ are absolute [5, pp. 1644-1645].

Finally

$$
I_{k}\left(-4,0 ; \frac{1}{2}\right)=\frac{\sqrt{\pi}}{2} \frac{\Gamma\left(\frac{k}{2}-\frac{1}{4}\right)^{2}}{\Gamma(k)}
$$

$[5$, formula $(5)]$.

Choosing $\epsilon^{\prime}$ in (3) small enough, we then see combining (3), (4) and (5) that the right-hand side of $(2)$ is

$$
\begin{gathered}
<<\frac{2^{3 k} \pi^{k}(k-1)}{\Gamma\left(k-\frac{1}{2}\right)}\left(\frac{\Gamma\left(k-\frac{1}{2}\right)^{2}}{2^{k} \Gamma(k)^{2}}+\frac{\Gamma\left(\frac{k}{2}-\frac{1}{4}\right)^{2}}{\Gamma(k)}+\frac{\Gamma\left(k-\frac{1}{2}\right)}{2^{k} \Gamma(k)}+\frac{\Gamma^{\prime}\left(k-\frac{1}{2}\right)}{2^{k} \Gamma(k)}\right) \\
=\frac{(4 \pi)^{k}(k-1)}{\Gamma(k)}\left(\frac{\Gamma\left(k-\frac{1}{2}\right)}{\Gamma(k)}+\frac{\Gamma\left(\frac{k}{2}-\frac{1}{4}\right)^{2} 2^{k}}{\Gamma\left(k-\frac{1}{2}\right)}+1+\frac{\Gamma^{\prime}\left(k-\frac{1}{2}\right)}{\Gamma\left(k-\frac{1}{2}\right)}\right)
\end{gathered}
$$

where the constant in $<<$ is absolute.

By Legendre's duplication formula one has

$$
2^{k-3 / 2} \Gamma\left(\frac{k}{2}-\frac{1}{4}\right)=\sqrt{\pi} \frac{\Gamma\left(k-\frac{1}{2}\right)}{\Gamma\left(\frac{k}{2}+\frac{1}{4}\right)} .
$$

Also

$$
\frac{\Gamma^{\prime}\left(k-\frac{1}{2}\right)}{\Gamma\left(k-\frac{1}{2}\right)}=\log \left(k-\frac{1}{2}\right)+\mathcal{O}\left(\frac{1}{k}\right) \quad(k \rightarrow \infty)
$$


$[1,6.3 .18]$.

Therefore we see that

(6)

$$
\sum_{f \in \mathcal{F}_{k}} \frac{D_{f}\left(k-\frac{1}{2}\right)}{\|f\|^{2}}<<\frac{(4 \pi)^{k}(k-1)}{\Gamma(k)}\left(\frac{\Gamma\left(k-\frac{1}{2}\right)}{\Gamma(k)}+\frac{\Gamma\left(\frac{k}{2}-\frac{1}{4}\right)}{\Gamma\left(\frac{k}{2}+\frac{1}{4}\right)}+\log k\right) .
$$

Observe that

$$
\frac{\Gamma(x+a)}{\Gamma(x+b)} \sim x^{a-b} \quad(x \rightarrow \infty)
$$

for any fixed real numbers $a$ and $b$ as follows immediately from Stirling's formula.

Using (7) on the right-hand side of (6) with $a=-\frac{1}{4}, b=\frac{1}{4}$ and $x=k-\frac{1}{4}$ resp. $x=\frac{k}{2}$ we therefore finally obtain that

$$
\sum_{f \in \mathcal{F}_{k}} \frac{D_{f}\left(k-\frac{1}{2}\right)}{\|f\|^{2}}<<\frac{(4 \pi)^{k}(k-1)}{\Gamma(k)}\left(\frac{1}{\sqrt{k}}+\log k\right) .
$$

On the other hand, given $\epsilon>0$, from [4] we infer that

$$
\|f\|^{2}<<_{\epsilon} \frac{\Gamma(k) k^{\epsilon}}{(4 \pi)^{k}}
$$

for all $f \in \mathcal{F}_{k}$ where the implied constant depends only on $\epsilon$ and not on $k$ (cf. the comments made in [8] and [6, sect. 3]).

We now use our assumption that $D_{f}\left(k-\frac{1}{2}\right) \geq 0$ for all $f \in \mathcal{F}_{k}$ and all $k$. Inserting (9) into (8) we then obtain

$$
\sum_{f \in \mathcal{F}_{k}} D_{f}\left(k-\frac{1}{2}\right)<<_{\epsilon} k^{1+\epsilon} \quad(k \rightarrow \infty)
$$

for any given $\epsilon>0$ as claimed.

\section{REFERENCES}

[1] M. Abramowitz and I. Stegun, Handbook of mathematical functions, Dover, New York, 1965.

[2] T. Apostol, Introduction to Analytic Number Theory, Springer, BerlinHeidelberg-New York, 1976.

[3] S. Gelbart and H. Jacquet, A relation between automorphic representations of $G L_{2}$ and $G L_{3}$, Ann. Sci. École Normale Sup. $4^{e}$ série, 11 (1978), 471-552. 
[4] H. Iwaniec, Small eigenvalues of Laplacian for $\Gamma_{0}(N)$, Acta Arith., 16 (1990), 65-82.

[5] W. Kohnen and J. Sengupta, Nonvanishing of symmetric square L-functions of cusp forms inside the critical strip, Proc. Amer. Math. Soc., 128, no. 6 (2000), 1641-1646.

[6] W. Kohnen and J. Sengupta, On quadratic character twists of Hecke L-functions attached to cusp forms of varying weights at the central point, Acta Arith., XCIX.1 (2001), 61-66.

[7] P. Sarnak, L-functions. Documenta Mathematica, extra vol. ICM 1998, I pp. 453-465.

[8] J. Sengupta, The central critical value of automorphic L-functions, C.R. Math. Rep. Acad. Sci. Canada, 22 (2) (2000), 82-85.

[9] G. Shimura, On the holomorphy of certain Dirichlet series, Proc. London Math. Soc., 31 (1975), 75-98.

[10] D. Zagier, Modular forms whose Fourier coefficients involve zeta-functions of quadratic fields, In: Modular Functions of One variable VI (eds. J.-P. Serre and D. Zagier), pp. 105-169, LNM no. 627, Springer, Berlin-Heidelberg-New York, 1976.

Winfried Kohnen

Universität Heidelberg

Mathematisches Institut

INF 288

D-69120 Heidelberg

Germany

winfried@mathi.uni-heidelberg.de

Jyoti Sengupta

T.I.F.R.

School of Mathematics

Homi Bhabha Road

400005 Bombay

India

sengupta@math.tifr.res.in 